\title{
Obtaining single-cycle pulses from a mode-locked laser
}

\author{
Victor V. Kozlov,,$^{1,2}{ }^{*}$ Nikolay N. Rosanov, ${ }^{3,4}$ and Stefan Wabnitz ${ }^{1}$ \\ ${ }^{1}$ Department of Information Engineering, Universitaá di Brescia, Via Branze 38, I-25123 Brescia, Italy \\ ${ }^{2}$ Physics Department, St. Petersburg State University, Petrodvoretz, St. Petersburg 198504, Russia \\ ${ }^{3}$ Institute of Laser Physics, Vavilov State Optical Institute, Birzhevaya liniya, 12, St. Petersburg 199034, Russia \\ ${ }^{4}$ St. Petersburg State University of Information Technologies, Mechanics and Optics, St. Petersburg 197101, Russia
}

(Received 18 July 2011; published 4 November 2011)

\begin{abstract}
In all existing mode-locked lasers, the ultimate limit on the output pulse duration is set by the bandwidth of the gain medium. Yet the technique of coherent mode locking allows one to generate broadband pulses from a laser with a linearly narrowband active medium by exploiting the coherent properties of the amplifier. In this work we numerically demonstrate how to use this technique for the generation of single-cycle pulses directly from a mode-locked oscillator.
\end{abstract}

DOI: 10.1103/PhysRevA.84.053810

PACS number(s): 42.60.Fc, 42.65.Re

\section{INTRODUCTION}

The bandwidth of ultrafast pulses generated by all types of conventional mode-locked lasers [1-3] is ultimately limited by the spectral width of the gain medium. This observation has driven, throughout the entire history of ultrafast lasers, the quest for the development of broadband laser materials. This activity culminated with the emergence of the Kerr-lens modelocked ultrabroadband Ti:sapphire laser [4], the workhorse of contemporary laser physics. Nevertheless, in spite of its enormous gain bandwidth, this laser is not able to generate pulses with durations shorter than two carrier frequency cycles [5-7]. We suggest an approach which differs drastically from the common trend, whereby we may use narrowband media for producing ultrawideband pulses by employing the technique of coherent mode locking (CML) [8]. Here we probe the extreme limit of this technique by demonstrating the proof-of-principle possibility of generating single-cycle pulses directly from a laser.

\section{PRINCIPLES OF OPERATION}

The main component of a laser mode locked by CML is the nonlinear coherent gain medium. As a matter of fact, the circulating pulse should be powerful enough in order to substantially power broaden the two-level amplifier and short enough to interact with the medium in a coherent manner. Achieving the power-induced broadening of the gain medium is a key to success. Under such conditions, it is not the linear gain spectral width that limits the bandwidth of the generated pulses but the width of the nonlinearly modified gain spectrum. The higher the field intensity, the broader the spectrum of the generated pulse. Note that the nature of the nonlinearity which is induced in the coherent amplifier is not related to the usual intensity or energy-dependent saturation effects. In fact, the amplifier responds to the pulse amplitude and supports the formation of the so-called $\pi$ pulses [9-11]. These $\pi$ pulses fully extract the energy which is stored in the gain medium by transferring all two-level systems from the upper state all the way down to the ground state, thus changing the population

\footnotetext{
*victor.kozlov@ing.unibs.it
}

difference from +1 on the leading edge of the pulse to -1 at its trailing edge. Therefore, the $\pi$ pulses are amplified most efficiently, as they are able to extract from the medium at least twice more energy than that possible from laser amplifiers operating in the linear or in the incoherent regimes. Moreover, in contrast to incoherent laser media, the coherent amplifier provides a shaping as well as a compression mechanism for the pulse. Namely, the condition of keeping the area of the pulse equal to $\pi$ provides a temporal compression when its power grows larger.

Nevertheless, the coherent nonlinear amplifier alone is not able to sustain stable mode-locked laser operation. To this end a second necessary component should be included, providing a mechanism which favors the pulsed regime against continuous wave $(\mathrm{cw})$ laser operation. Such a component can be the active periodic modulation of intracavity losses, as in Refs. [12,13], or a passive saturable absorber, as suggested in Ref. [8], which is also the method of choice here. Basically, a continuous wave which is linearly interacting with all intracavity elements should experience net losses; at the same time, the nonlinear pulse sees a net gain. Such a discrimination can be achieved by placing a nonlinear absorber inside the cavity. This absorber must provide minimum losses for strong pulses and simultaneously provide high loss for $\mathrm{cw}$ radiation. The necessary difference in losses arises from the loss saturation effect which is induced by the intense pulse. Conventional passively mode-locked lasers use fast or slow (as far as the inversion recovery time when compared with the pulse duration) saturable absorbers (e.g., a two-level medium) whose polarization decay time always remains faster than the pulse duration. To the contrary, in the technique of CML we choose to incorporate a coherent absorber, i.e., an absorber having all its relaxation times shorter than the pulse duration. Such a choice is dictated by practical considerations; namely we can use for the nonlinear absorber the same medium that is used for the coherent nonlinear amplifier, the only difference being that the absorber is not externally pumped. In this way we naturally match the transition frequencies of the absorber and the amplifier, thus avoiding the usual problem of finding an appropriate pair of transitions for the amplifier and the absorber.

In order to provide the condition of minimum losses experienced by the pulse in the intracavity absorber, which 
is necessary for the stable operation of the laser, one should operate in the regime of self-induced transparency [14,15]. In this regime, a strong pulse transfers all the population of the absorbing two-level medium from its ground state into the upper state on the leading front. Next, on the trailing edge, the pulse brings all the population back from the upper state into the ground state by the process of stimulated emission. Ideally (for infinitely long relaxation times) there is no energy left in the absorber after the pulse, so the net losses for the $2 \pi$ pulse are equal to zero. Such self-induced transparency absorber acts as a pulse shaper. This shaping mechanism is not particularly important for achieving CML, as the amplifier alone introduces a sufficient pulse shaping mechanism. In fact, in perspective, we may also think of a CML configuration in which the coherent absorber is replaced by an incoherent fast or slow absorber. In such a case we would still observe the key feature of CML_-generation of pulses with bandwidth far beyond the linear gain spectral width. Here we use a coherent absorber for the purpose of providing small losses for the nonlinear self-induced transparency pulse, while the losses for linear continuous wave radiation are maintained at a much higher level, as they follow classical Beer's decay law (for quantitative stability analysis see Ref. [8]).

The condition that the pulse circulating inside the cavity is, at the same time, a $\pi$ pulse in the amplifier and a $2 \pi$ pulse in the absorber is particularly easy to implement in a cavity where the two media are spatially separated. Simply, the spatial cross section of the beam in the absorber should be twice as small as that in the amplifier. Alternatively, the use of semiconductor materials may permit to grow periodic structures involving alternating amplifier and absorber layers with a predetermined (in our case twice) difference of dipole moments. In this way, both absorbing and amplifying media encounter the same beam cross section, as was suggested for the case of quantum cascade lasers in Ref. [16]. Note that CML lasers enjoy the practically important property that their operation is not disrupted even in the presence of relatively large departures of the pulse area from the $\pi$ and $2 \pi$ conditions, as it will also be confirmed by the present analysis.

The larger the intensity of the intracavity pulse in the CML laser, the shorter its duration (and, correspondingly, the broader its spectrum). Ideally, the pulse from a CML laser is chirp free, i.e., transform limited. The spectral width of the emitted pulse is broader than the gain spectral width: In practice, the pulse spectral width is limited only by the achievable intracavity power. More precisely, the pulse spectral width is determined only by the value of its Rabi frequency $\Omega_{R}$, i.e., the product of the atomic transition dipole moment $d_{p}$ and the strength of the electric field $E$ divided by the Planck constant $\hbar$ : $\Omega_{R}=d_{p} E / \hbar$. As a consequence, the necessary intracavity power is reduced for media with relatively large dipole moments. The concept of CML is particularly relevant when considering semiconductor materials; whereas this technique is hardly applicable in practice to solid-state lasers, as, in this case, the generation of femtosecond $\pi$ pulses would require prohibitively large optical peak powers. Given that the intracavity Rabi frequency is regulated by a balance among the achievable gain and intracavity losses, achieving the $\pi$ condition for the pulse area automatically determines the pulse duration $\tau_{p}$. Therefore, we may write $\tau_{p} \approx \Omega_{R}^{-1}$.

\section{MODEL AND RESULTS}

Given that a sufficient intracavity power can be achieved, no particular limitation to the pulse duration follows from the theory of CML, the only natural limit being the inverse frequency of the two-level transition. Indeed, in this article we show how this limit can be reached, thereby approaching the attosecond range of durations. In this extreme situation the approximations that are usual for conventional mode-locked lasers break down, so we have to abandon the slowly varying, rotating-wave, and unidirectional propagation approximations altogether. The two Maxwell equations

$$
\frac{\partial D}{c \partial t}-\frac{\partial H}{\partial z}=0 \quad \text { and } \quad \frac{\partial H}{c \partial t}-\frac{\partial E}{\partial z}=0
$$

for the electric $E$ and magnetic $H=B$ fields are coupled to two systems of nonreduced Bloch equations. The first of such systems describes the homogeneously broadened amplifier:

$$
\begin{gathered}
\frac{\partial}{\partial t} \rho_{21}^{(a)}=-\left(i \omega_{21}^{(a)}+\gamma_{21}^{(a)}\right) \rho_{21}^{(a)}-i \frac{d_{a} E}{\hbar}\left(\rho_{22}^{(a)}-\rho_{11}^{(a)}\right), \\
\frac{\partial}{\partial t} \rho_{22}^{(a)}=-\gamma_{2}^{(a)} \rho_{22}^{(a)}-i \frac{d_{a} E}{\hbar}\left(\rho_{21}^{(a)}-\rho_{12}^{(a)}\right)+p, \\
\frac{\partial}{\partial t} \rho_{11}^{(a)}=\gamma_{2}^{(a)} \rho_{22}^{(a)}-\gamma_{1}^{(a)} \rho_{11}^{(a)}+i \frac{d_{a} E}{\hbar}\left(\rho_{21}^{(a)}-\rho_{12}^{(a)}\right),
\end{gathered}
$$

and the second system is associated with the homogeneously broadened absorber:

$$
\begin{gathered}
\frac{\partial}{\partial t} \rho_{21}^{(p)}=-\left(i \omega_{21}^{(p)}+\gamma_{21}^{(p)}\right) \rho_{21}^{(p)}-i \frac{d_{p} E}{\hbar}\left(\rho_{22}^{(p)}-\rho_{11}^{(p)}\right), \\
\frac{\partial}{\partial t} \rho_{22}^{(p)}=-\gamma_{2}^{(p)} \rho_{22}^{(p)}-i \frac{d_{p} E}{\hbar}\left(\rho_{21}^{(p)}-\rho_{12}^{(p)}\right), \\
\frac{\partial}{\partial t} \rho_{11}^{(p)}=\gamma_{2}^{(p)} \rho_{22}^{(p)}+i \frac{d_{p} E}{\hbar}\left(\rho_{21}^{(p)}-\rho_{12}^{(p)}\right) .
\end{gathered}
$$

Here $D=E+4 \pi P$ with the polarization $P=$ $N_{p} d_{p} \rho_{12}^{(p)}+N_{a} d_{a} \rho_{12}^{(a)}+$ c.c.; $N_{p, a}$ are the concentrations of passive (absorber) and active (amplifier) twolevel systems; $d_{p, a}$ are the (real) dipole matrix elements of the transitions between upper (2) and lower (1) states; $\omega_{12}^{(a, p)}$ are the transition frequencies, which are taken to be equal to each other, as well as to the carrier frequency $\omega_{0}$ of the seed pulse which triggers the mode-locking operation; $\gamma_{21}^{(a, p)}, \gamma_{2}^{(a, p)}$, and $\gamma_{1}^{(a)}$ are the relaxation rates with obvious meanings; and, finally, $p$ is the pump rate. Note that the absorber is modeled by a closed system, which implies a conservation law in the form $\rho_{11}^{(p)}+\rho_{22}^{(p)}=1$, whereas the laser amplifier is modeled as usual by an open system, for which a similar conservation law does not hold. Note that in the framework of a similar model (but with inclusion of the Drude conductivity) we demonstrated in Ref. [17] the generation of few-cycle Maxwell-Drude-Bloch dissipative solitons in the single-pass propagation regime. The mechanism leading to the formation of these solitons can be considered a prerequisite for the existence of a stable mode-locked regime for the CML laser.

In our numerical simulations we used the following set of parameters: the ratio of dipole moments $\sqrt{\mu}=d_{p} / d_{a}$ was 1.5 . Such a value was intentionally made to differ from the ideal case of 2 , in order to test the relative insensitivity 


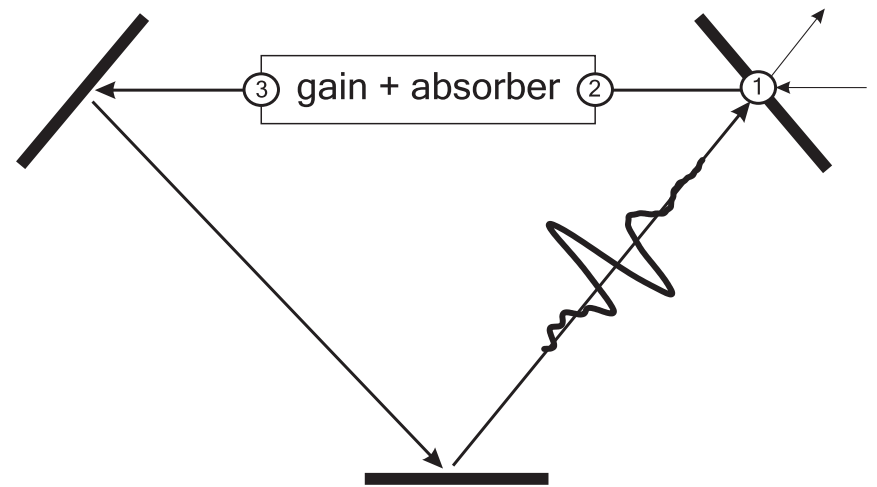

FIG. 1. Schematic of the coherently mode-locked laser. Amplifier and absorber are implemented within the same sample, as used in the numerical model. If necessary, the gain and absorbing media can be placed in different locations inside the cavity.

of the CML operation to the precise value of this ratio, which is important for the practical implementation of the present technique. The relaxation rates were $\gamma_{1}^{(a)}=0.025$, $\gamma_{2}^{(a)}=0.005, \quad \gamma_{2}^{(p)}=0.006, \quad \gamma_{21}^{(p)}=0.0025, \quad \gamma_{21}^{(a)}=0.015$, and the pump rate was $p=0.004$. All of these quantities are expressed in units of the frequency $\omega_{12}^{(a)}=\omega_{12}^{(p)}$. Other parameters are chosen in such a way that the coupling constants between the field (which is expressed in terms of the dimensionless Rabi frequency $\Omega=\Omega_{R} / \omega_{12}^{(p)}$ ) and the polarizations induced by the passive and active systems read as $\beta=4 \pi N_{p} d_{p}^{2} / \hbar \omega_{21}^{(p)}=0.1$ and $\kappa=4 \pi N_{a} d_{p} d_{a} / \hbar \omega_{21}^{(a)}=0.02$. As an estimate for the intracavity intensity $I$ we can write $I=\left(104 / x^{2}\right) \mathrm{GW} / \mathrm{cm}^{2}$ for $\Omega_{R}=\omega_{12}^{(a)}=10^{15} \mathrm{~s}^{-1}$ where $d_{p}=1.6 x \times 10^{-28} \mathrm{Cm}$; here the spatial extension of the dipole $x$ is expressed in nanometers. Thus, for $x=10$ the intracavity intensity becomes as small as $1 \mathrm{GW} / \mathrm{cm}^{2}$.

The model Eqs. (1)-(7) need to be supplemented by appropriate boundary conditions. We considered a ring-cavity configuration supporting predominantly unidirectional propagation, consisting of a number of mirrors, one of which is partially transparent with the amplitude reflection coefficient $R$; see Fig. 1 . In this case, the relation $\Omega_{\mathrm{ref}}=R \Omega_{\text {in }}$ establishes the connection between the wave which is incident on the mirror $\Omega_{\text {in }}$ and the reflected wave $\Omega_{\text {ref }}$. The balance between the gain $\kappa$ and the mirror out-coupling losses $(1-R)$ provides a key relation in our model, as it ultimately determines the intracavity pulse power. In order to generate pulses of one carrier period in duration, i.e., with $\tau_{p} \approx \omega_{12}^{-1}$, we need to ensure the large gain-to-loss ratio which permits us to reach the condition $\Omega \approx 1$. The next important parameter is the strength of the absorber $\beta$ : its large value is necessary for the stability of the mode-locked operation. On the other hand, the precise values of the various relaxation constants are not that important. The only requirement here is that the total length $L$ of the cavity is long enough to guarantee a full recovery of the equilibrium states of the amplifier and the absorber before the pulse comes back after a round-trip through the cavity.

We performed numerical simulations of the approximationfree Maxwell-Bloch equations based on the finite-difference time-domain integration method. The numerics mimic the
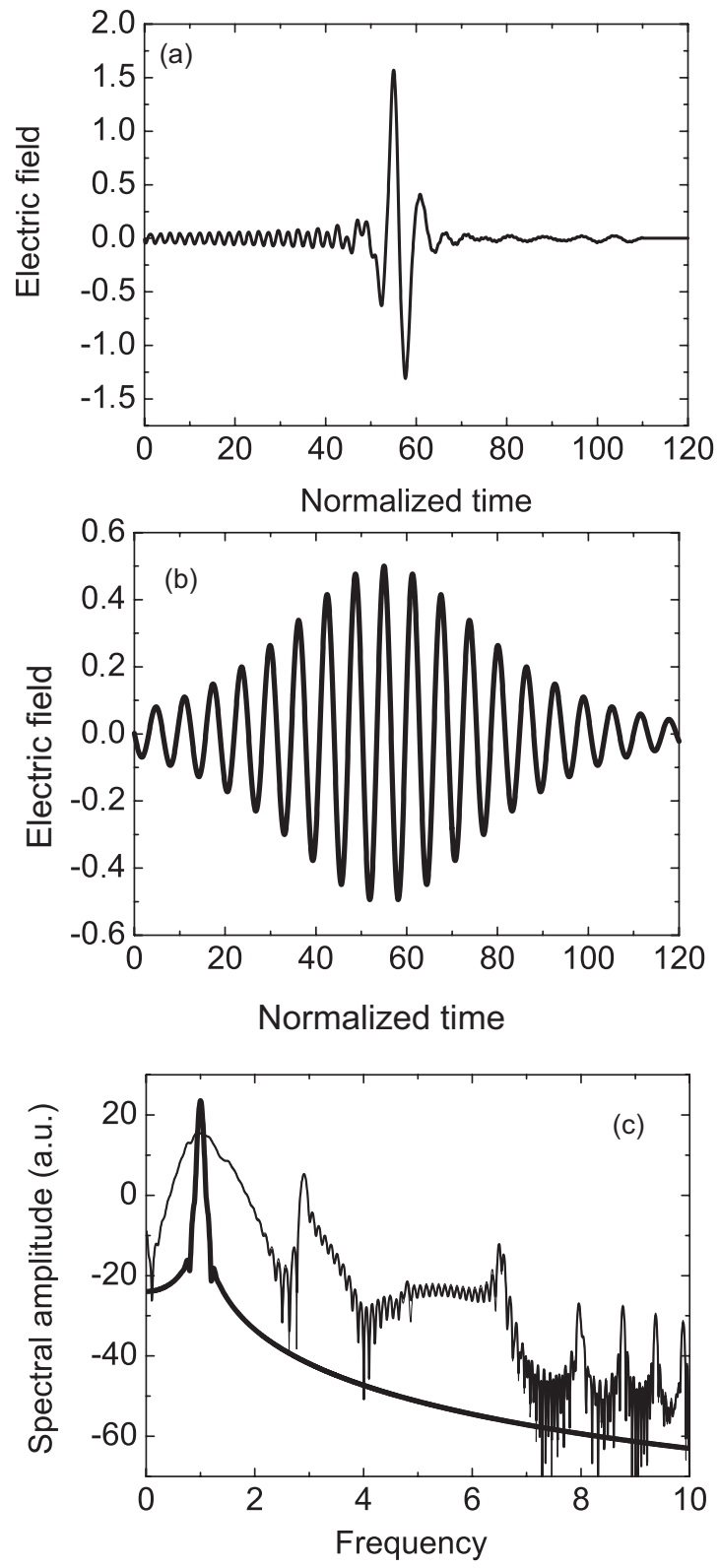

FIG. 2. Electric field and its spectrum. (a) Electric field in the steady-state regime (after 130 round-trips) at the out-coupling mirror (at point 1 in Fig. 1). (b) The seed (input) pulse is shown. (c) Spectrum of the pulse from (a) (medium thin line), spectrum of the seed pulse (thick line), spectrum of the pulse after 75 round-trips (thin line). Spectra of the pulses after 75 and 130 round-trips are almost undistinguishable, demonstrating that a true steady state is achieved. The spectral amplitude of the pulse on the vertical axis is defined as $20 \ln (\sqrt{F(\omega)})$, where $F(\omega)$ is the $\omega$ component of the Fourier power spectrum. Variables on the vertical and horizontal axes are both dimensional: time and frequency are normalized to $\omega_{12}$; electric field is the normalized Rabi frequency $d_{p} E / \hbar \omega_{21} . R=0.92$, other parameters are as explained in the text.

intracavity dynamics of the pulse circulating between mirrors: starting from point 1 in Fig. 1, the seed pulse which is shown in Fig. 2(b) experiences free propagation in vacuum until point 2 . The pulse next experiences gain and shaping in the amplifier as well as simultaneous absorption and shaping in the absorber until point 3 , where the pulse is again subjected 

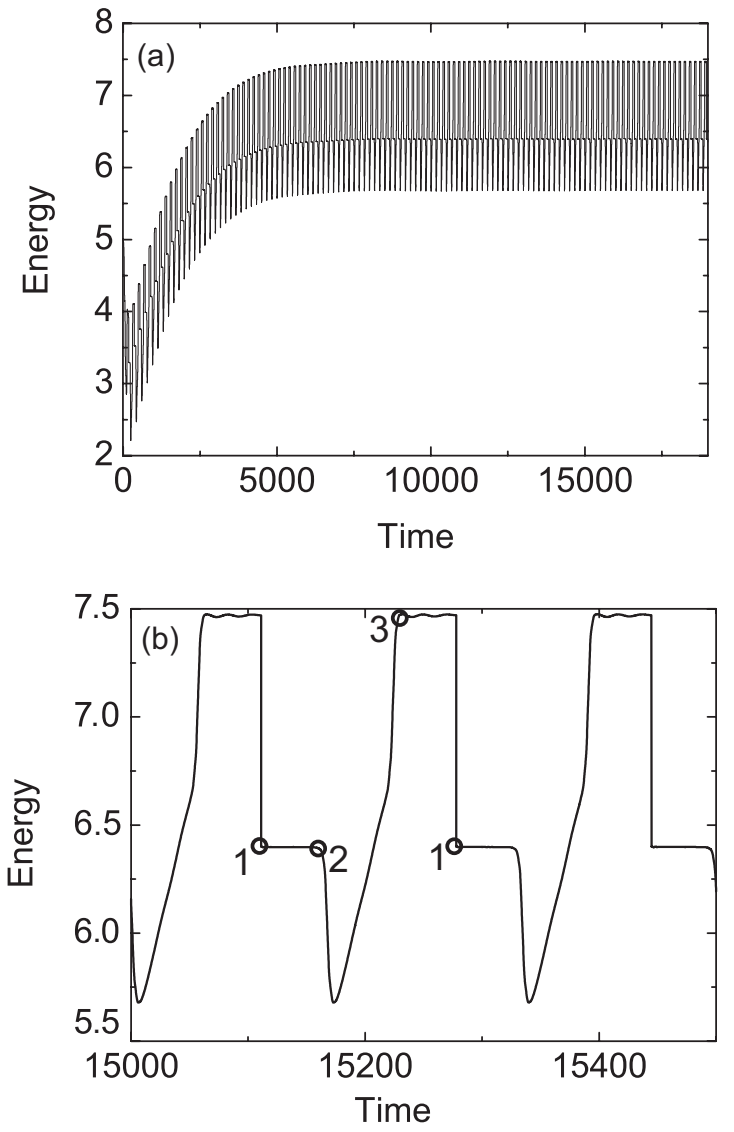

FIG. 3. Temporal evolution of the intracavity energy. Simulation conditions are as for Fig. 1. (b) An exploded view of three roundtrips. Numbers indicate those points in time when the pulse crosses the characteristic points in the cavity, as introduced in Fig. 1. $T$ is the round-trip time. Energy is the dimensionless quantity, namely $\omega_{12} \int_{-\infty}^{\infty} d t\left(d_{p} E / \hbar \omega_{21}\right)^{2}$.

to free propagation until point 1 , where the circulating pulse experiences partial transmission at the out-coupling mirror. A full round-trip is thus completed. Fewer than 100 round-trips are needed in order to reach the stead-state shape which is shown in Fig. 2. Additional losses appear as a result of reflection of the pulse from the boundary between vacuum and the resonant media. The back-propagating wave which is generated at each round-trip rapidly vanishes since the laser supports only unidirectional beam propagation.

The CML laser oscillation does not start from spontaneous noise since the cavity is lossy for low-intensity $\mathrm{cw}$ radiation. Therefore, a relatively long and powerful many-cycle femtosecond pulse (as it is shown in Fig. 2(b)) was used in order to initiate the mode-locked operation. Many computer runs performed with different values of $R$ and started from this seed pulse demonstrated a $10 \div 20$-fold temporal compression, accompanied by pulse evolution toward a steady-state regime involving the generation of a recirculating pulse of about one cycle in duration. The corresponding evolution of the pulse energy is shown in Fig. 3. This figure shows that large variations of the pulse energy occur in the course of its sequential passing through the various intracavity elements. The onset of a steady state is clearly seen in the figure, since the pulse dynamics converges to a perfectly periodic regime, with a period equal to the round-trip time.

For $R=0.95$ we obtained (not shown here) a steady-state pulse that was 1.5 times shorter than the pulse of Fig. 2; this pulse was accompanied by a well-pronounced precursor. Such a precursor represents the third-order harmonic of the main pulse carrier frequency: The precursor spectral shape is larger in intensity and narrower in width than the main spectral component. If necessary, the precursor can be filtered away by a spectral filter, thus yielding a clean one-cycle pulse.

\section{CONCLUSIONS}

We intentionally limited ourselves to consider the minimal generic model, in which one-dimensional geometry is justified by a proper choice of single-transverse-mode cavity or waveguide without cutoff frequency. Also, the effects of dispersive broadening by various intracavity elements, as well as the possible presence of extra nonlinearities should be addressed separately. For instance, dispersion control could be achieved by using chirped multilayer mirrors with a transparency region wider than $500 \mathrm{~nm}$ [18]. In general, problems associated with practical implementation of a few-cycle pulse laser are common also to other studies in this field; see, for example, Refs. [19-21]. The additional possible presence of infrared and/or ultraviolet absorption bands, as well as the inhomogeneous broadening which is characteristic of self-assembled quantum dots, may also be added to our model, as it was shown for the generation of few-cycle pulses in Ref. [22]. Instead of a seed pulse triggering the generation, we may think of using gain switching $[23,24]$, i.e., the modulation of the electric current in the semiconductor amplifier.

\section{ACKNOWLEDGMENTS}

This work was carried out with support from the Cariplo Foundation Grant No. 2009-2730. N.N.R. acknowledges the Cariplo Foundation grant of the Landau Network, Centro Volta, for the support of his work at the Università degli Studi di Brescia, as well as Grant No. 2.1.1/9824 of the Russian Ministry of Education and Science and Grant No. 11-0212250-ofi-m of the Russian Foundation for Basic Research.
[1] P. M. W. French, Rep. Prog. Phys. 58, 169 (1995).

[2] H. A. Haus, IEEE J. Sel. Top. Quantum Electron. 6, 1173 (2000).

[3] U. Keller, Nature 424, 831 (2003).

[4] D. E. Spence, P. N. Kean, and W. Sibbett, Opt. Lett. 16, 42 (1991).
[5] U. Morgner et al., Opt. Lett. 24, 411 (1999).

[6] D. H. Sutter et al., Opt. Lett. 24, 631 (1999).

[7] R. Ell et al., Opt. Lett. 26, 373 (2001).

[8] V. V. Kozlov, Phys. Rev. A 56, 1607 (1997).

[9] J. P. Wittke and P. J. Warter, J. Appl. Phys. 35, 1668 (1964). 
[10] A. Icsevgi and W. E. Lamb Jr. Phys. Rev. 185, 517 (1969).

[11] F. A. Hopf and M. O. Scully, Phys. Rev. 179, 399 (1969).

[12] J. M. Dudley, J. D. Harvey, and R. Leonhardt, J. Opt. Soc. Am. B 10, 840 (1993).

[13] P. D. Drummond, J. D. Harvey, J. M. Dudley, D. B. Hirst, and S. J. Carter, Phys. Rev. Lett. 78, 836 (1997).

[14] S. L. McCall and E. L. Hahn, Phys. Rev. Lett. 18, 908 (1967).

[15] S. L. McCall and E. L. Hahn, Phys. Rev. 183, 457 (1969).

[16] C. R. Menyuk and M. A. Talukder, Phys. Rev. Lett. 102, 023903 (2009).

[17] N. N. Rosanov, V. V. Kozlov, and S. Wabnitz, Phys. Rev. A 81, 043815 (2010).
[18] T. Brabec and F. Krausz, Rev. Mod. Phys. 72, 545 (2000).

[19] H. Leblond, H. Triki, and D. Mihalache, Phys. Rev. A 84, 023833 (2011).

[20] E. Goulielmakis et al., Science 320, 1614 (2008).

[21] G. Krauss, S. Lohss, T. Hanke, A. Sell, S. Eggert, R. Huber, and A. Leitenstorfer, Nat. Photon. 4, 33 (2010).

[22] N. V. Vysotina, N. N. Rosanov, and V. E. Semenov, Opt. Spectr. 106, 713 (2009).

[23] J. AuYeung, Appl. Phys. Lett. 38, 308 (1981).

[24] T. Sogawa, Y. Arakawa, M. Tanaka, and H. Sakaki, Appl. Phys. Lett. 53, 1580 (1988). 